\title{
Nitroxide radical attenuates ischaemia/reperfusion injury to the rat small intestine
}

\author{
R Udassin, Y Haskel, A Samuni
}

\begin{abstract}
Background-Free radicals are associated with post-ischaemic intestinal injury and contribute to major clinical problems primarily in premature infants. Various antioxidative means and modes of intervention, previously tested, have demonstrated only limited efficacy.

Aims-To study the protective activity of the stable nitroxide radical 4-OH, 2,2,6,6tetramethylpiperidine-1-oxyl (TPL) and its respective hydroxylamine (TPL-H) against ischaemia/reperfusion (I/R) injury. Methods-An isolated loop of ileum was created in laboratory male Sabra rats and constantly perfused with warmed normal saline. Intestinal injury was elicited through clamping of the superior mesenteric rat artery followed by reperfusion. Either TPL or TPL-H was given intravenously immediately before ischaemia or reperfusion and continuously afterwards. The rate of mucosal to lumen clearance of para-aminohippurate (PAH) was used to evaluate intestinal mucosal injury. Serum and perfusate levels of both TPL and TPL-H were measured using electron paramagnetic resonance spectrometry.

Results-The increase in intestinal permeability induced by $I / R$ was significantly inhibited by both TPL and TPL-H. The nitroxide was effective also when given immediately before reperfusion.

Conclusion-Through a continuous exchange, TPL and TPL-H act as selfreplenishing antioxidants and thus protect from intestinal injury. This demonstrates the potential of the family of nitroxide antioxidants against oxidative stress in general and $I / R$ injury in particular.

(Gut 1998;42:623-627)
\end{abstract}

Pediatric Surgery

Hadassah University

Hospital Mount

Scopus, Jerusalem,

Israel

R Udassin

Y Haskel

Department of Molecular Biology,

Hebrew

University-Hadassah

Medical School,

Jerusalem, Israel

A Samuni

Correspondence to:

Dr R Udassin.

Accepted for publication 9 October 1997 meability is one of the earliest physiological changes in intestinal I/R injury, ${ }^{6}$ the flux of plasma or luminal clearances of water soluble molecules has proved to be the most meaningful marker for evaluating injury. ${ }^{9-12}$

Intestinal ischaemia, in both acute and chronic forms, is a major clinical problem in adults as urther exacerbated in the reperfusion period. Studies of animal models of ischaemi reperfusion (I/R) have used histological ${ }^{7}$ and The ischaemic insult elicits damage, whic
Radical and non-radical reactive oxygen derived species (ROS) have been previously implicated in mediation of $I / R$ damage in general and intestinal injury in particular. Hence, the enzyme activities of tissue superoxide dismutase (SOD), catalase, or glutathione (GSH) reductase, as well as cellular levels of endogenous antioxidants such as GSH in animal experimental models of necrotising enterocolitis (NEC), were investigated. ${ }^{10}{ }^{13}$ Previous studies particularly focused on the activity of xanthine oxidase, which gives rise to $\mathrm{O}_{2}^{-}$and $\mathrm{H}_{2} \mathrm{O}_{2}$, employing specific inhibitors to limit their formation. ${ }^{1012}{ }^{14}{ }^{15}$ Various low molecular weight antioxidants (LMWA) including vitamin $\mathrm{E}$, the spin trap phenyl t-butyl nitrone, and antioxidative enzymes such as SOD and catalase have been previously tested in an attempt to provide protection from I/R injury. ${ }^{12}$ None of these agents significantly attenuated the increase in mucosal permeability caused by occlusion of the superior mesenteric artery for 10 minutes in six week old rats, which suggests that $\mathrm{I} / \mathrm{R}$ injury is not mediated by ROS. ${ }^{12}$

Recently the use of stable nitroxide radicals, which constitute a unique and unusual class of antioxidants, has been proposed as a complementary strategy for modulating oxidative injury. Contrary to exogenously added SOD or catalase and several common antioxidants, nitroxides readily cross the blood-brain barrier and permeate the cell membrane. The biological effect of nitroxides has been previously studied using diverse physical, chemical, biochemical, and cellular means of insult which initiate injurious processes mediated by ROS. The protective activity of nitroxides has been demonstrated against ethanol, ${ }^{16}$ non-steroidal anti-inflammatory drugs, ${ }^{16}$ gastric and colonic irritants such as trinitrobenzene sulphonate, ${ }^{17}$ mechanical trauma, ${ }^{18}$ and hyperbaric oxygen ${ }^{19}$ in rat experimental models. Additionally, because nitroxides are unreactive toward most diamagnetic species, but rather selectively react with free radicals without forming secondary radicals, they can serve also as a powerful research tool for probing the roles played by deleterious radicals.

By undergoing 1-electron transfer reactions, nitroxides are readily reduced in vivo to hydroxylamines or oxidised to oxo-ammonium cations. Consequently, all three forms can be present in the tissue as shown below for 4-OH2,2,6,6-tetramethylpiperidine-N-oxyl (TPL). The non-radical species might also comproportionate to yield the more stable radical form and thus the nitroxide can self-replenish itself. Consequently, unlike antioxidants which act in 
$\mathrm{TPL}^{+}$

oxo-ammonium cation

TPL

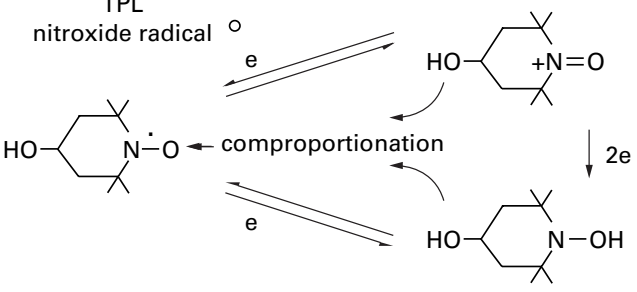

TPL-H

hydroxylamine

a sacrificial mode, nitroxide can provide protection in a catalytic way.

The present study concentrated on the potential use of nitroxide and its respective hydroxylamine as antioxidants for the modulation of $\mathrm{I} / \mathrm{R}$ intestinal injury.

\section{Methods}

All studies were performed using male Sabra rats weighing $180-220 \mathrm{~g}$ and randomly assigned to the various experimental groups. Experimental manipulations were performed in accordance with guidelines established by the Animal Welfare Committee (Hadassah University Hospital). Animals were housed in individual cages and given rat chow and water ad libitum. Food, but not water, was withdrawn 18 hours before study.

\section{ANIMAL PREPARATION}

The rats were anaesthetised with an intraperitoneal injection of $100 \mathrm{mg} / \mathrm{kg}$ body weight thiobarbital sodium salt (Inactin, Research Biomedical International, Natick, Massachusetts, USA). Following anaesthesia a tracheostomy was performed and the body temperature monitored by a rectal probe: when necessary, core temperature was maintained at $37^{\circ} \mathrm{C}$ by means of an infrared heating lamp. The right

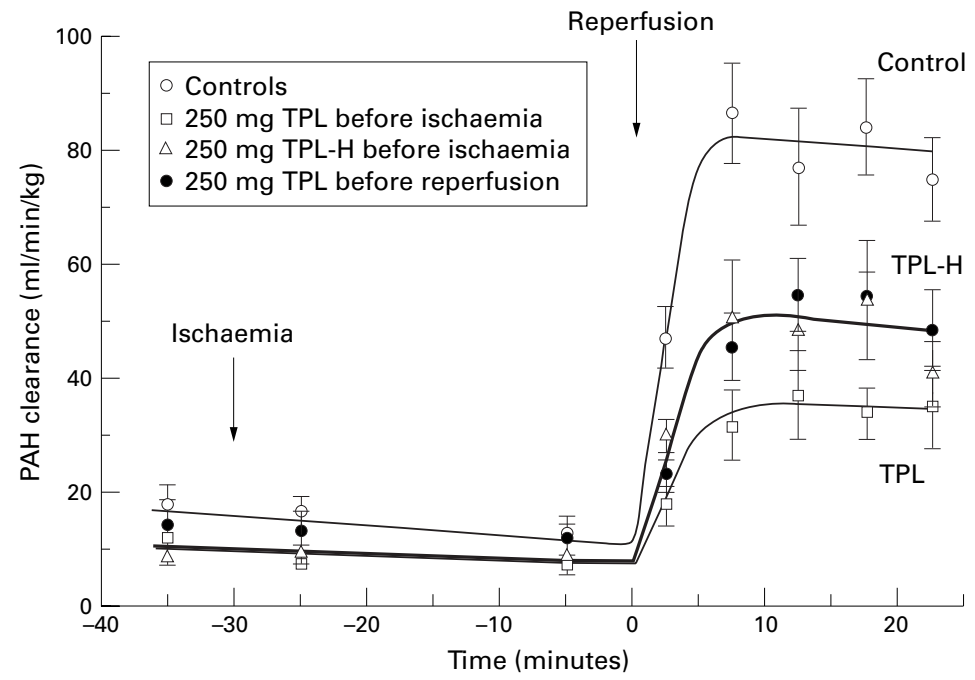

Figure 1 Effect of TPL and TPL-H on intestinal permeability induced by I/R. Mean (SE) mucosal to lumen clearance rate of $P A H$ in the eight periods of five minutes under evaluation: pre-ischaemia, ischaemia and five consecutive five minute periods of reperfusion in the various experimental groups. Before ischaemia the rats were treated intravenously with either saline as control, $250 \mathrm{mg} T P L / \mathrm{kg}$ given before ischaemia, $250 \mathrm{mg} T P L-H / \mathrm{kg}$ given before ischaemia, or $250 \mathrm{mg} T P L / \mathrm{kg}$ given immediately before reperfusion. carotid artery was cannulated and systemic arterial pressure was measured with a Srarhman P23A pressure transducer (Gould, Oxnard, California, USA) connected to the carotid artery cannula. Systemic blood pressure and heart rate were continuously monitored with a physiological recorder (Gross Instruments Co, Quincy, Massachusetts, USA). The right femoral vein was also cannulated for indicator injection and for drug administration. The abdomen was opened through a midline incision and the renal vessels were ligated to prevent urinary excretion of para-aminohippurate (PAH; Sigma A1422). A $10 \mathrm{~cm}$ distal ileal loop was isolated, cannulated at both proximal and distal ends, and flushed with warm normal saline solution. The intestine and abdominal contents were subsequently covered with plastic wrap to prevent evaporative water loss.

EXPERIMENTAL PROTOCOL

The cannulated ileal segment was perfused with warm $0.9 \% \mathrm{NaCl}$ solution at a rate of $1 \mathrm{ml} / \mathrm{min}$ until the effluent appeared clear. Then PAH $(58 \mathrm{mg} / \mathrm{kg})$ was intravenously administered followed by an equilibration period of 50 minutes. After an initial control collection the superior mesenteric artery was clamped for 15 minutes during which perfusate was continuously collected. After release of the clamp five consecutive perfusate collections of five minutes each were made with blood samples drawn at the mid points.

\section{EXPERIMENTAL GROUPS}

Three experimental groups were compared with the control. In two groups either TPL or TPL-H in saline as vehicle was given in an intravenous bolus of $250 \mathrm{mg} / \mathrm{kg}$ immediately before ischaemia and at a continuous rate of $3.3 \mathrm{mg} / \mathrm{kg} / \mathrm{min}$ throughout the ischaemia and reperfusion periods. In the third experimental group TPL was administered to the rat at the end of the ischaemic period and continuously during reperfusion. The control group consisted of rats which underwent the same experimental protocol but were given the vehicle alone at the same volume.

ELECTRON PARAMAGNETIC RESONANCE (EPR) MEASUREMENTS

To determine nitroxide concentration, blood and perfusate were sampled and kept frozen. Immediately after thawing the samples were drawn into a gas permeable, $0.8 \mathrm{~mm}$ inner diameter, Teflon capillary. The capillary was inserted into a quartz tube open at both sides and then placed within the EPR spectrometer cavity. The EPR spectra were recorded on a JEOL JES-RE3X ESR spectrometer working at $\mathrm{X}$ band with centre field set at $3362 \mathrm{G}$, $100 \mathrm{kHz}$ modulation frequency, $1 \mathrm{G}$ modulation amplitude, $0.4 \mathrm{~mW}$ incident microwave power. The concentration of TPL was calculated from the EPR signal intensity using nitroxide standard solution. To determine the total level of TPL and TPL-H, $1 \mathrm{mM}$ $\mathrm{K}_{3} \mathrm{Fe}(\mathrm{CN})_{6}$ was added to oxidise TPL-H to TPL and the EPR signal was measured again. 
PAH DETERMINATION

For determination of PAH clearance, the method according to Brun $^{20}$ was adapted for small volumes. One $\mathrm{ml}$ of the perfusate liquid was added to $100 \mu 110 \%$ TCA. Samples of $300 \mu \mathrm{l}$ whole blood were mixed with $1200 \mu \mathrm{l}$ $10 \%$ TCA for deproteinisation, cooled to $4^{\circ} \mathrm{C}$ for 20 minutes, centrifuged, separated, and the supernatant kept at $-70^{\circ} \mathrm{C}$. For spectrophotometric (Cobos Bio, Roche, Basel, Switzerland) $\mathrm{PAH}$ measurement at $465 \mathrm{~nm}$, $100 \mu \mathrm{l}$ of Ehrlich's reagent ( $1 \mathrm{~g} p$-dimethylaminobenzaldehyde (Sigma D2004), $60 \mathrm{ml}$ alcohol $93 \%, 4 \mathrm{ml} 2 \mathrm{~N} \mathrm{HCl}$, distilled water to a final total volume of $100 \mathrm{ml}$ ) was added to a $50 \mu \mathrm{l}$ sample and $30 \mu \mathrm{l}$ distilled water. Standards ranged from 0.1 to $1.0 \mathrm{mg} \%$.

CALCULATION OF PAH CLEARANCE

The plasma to lumen clearance of $\mathrm{PAH}$ was calculated as follows:

$$
\text { Clearance }=\frac{\begin{array}{c}
\text { Perfusate }(\mathrm{U} / \mathrm{mL}) \times \\
\text { Perfusation rate }
\end{array}}{\text { Plasma }(\mathrm{U} / \mathrm{mL}) \times}
$$

where clearance is expressed in $\mathrm{ml} / \mathrm{min} / \mathrm{kg}$.

ANALYSIS OF DATA

Differences within the groups were analysed using analysis of variance by ranks (MannWhitney test). The null hypothesis was rejected at $\mathrm{p}<0.05$ using a two tailed test.
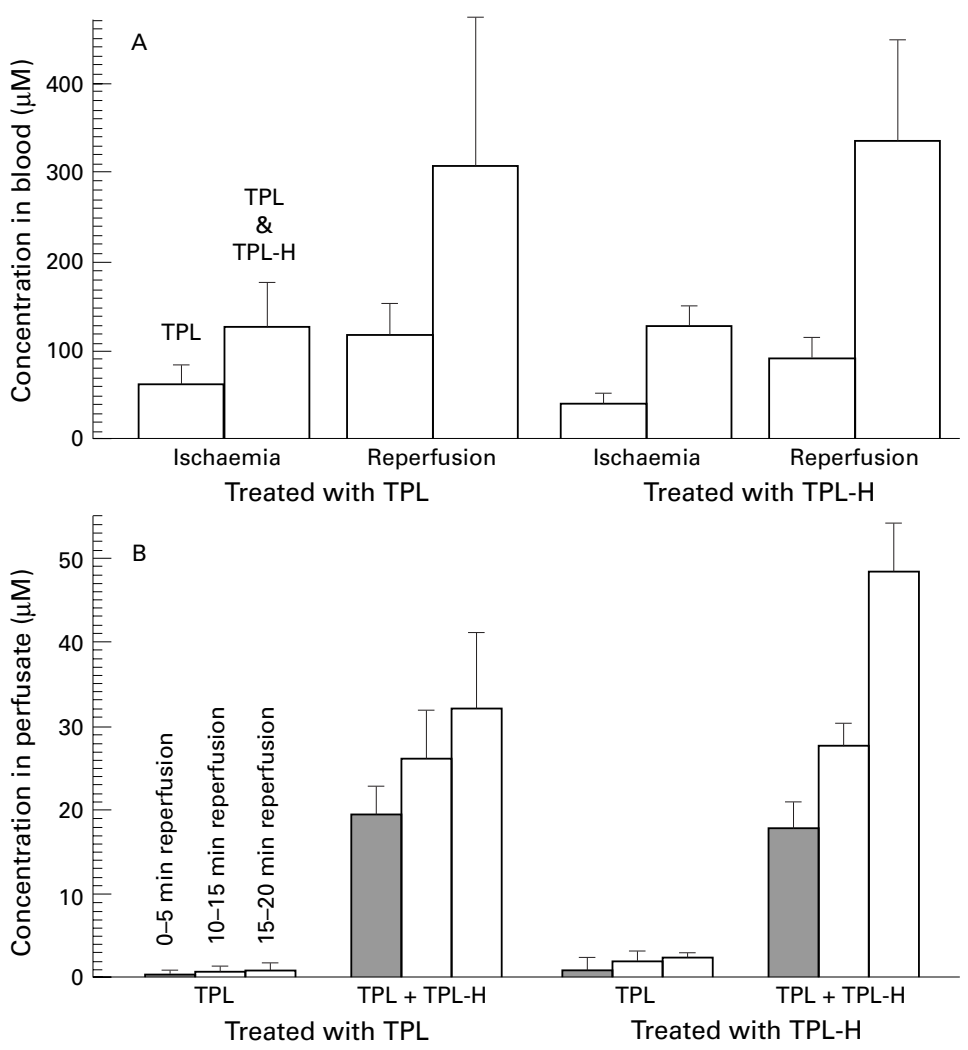

Figure 2. Distribution of TPL and TPL-H in (A) blood and (B) perfusate during ischaemia and reperfusion. Aliquots of blood and perfusate were sampled during ischaemia and reperfusion of rats treated intravenously with either TPL or TPL-H and scanned for the EPR signal as detailed in the Methods section. $K_{3} \mathrm{Fe}(\mathrm{CN})_{6} 1 \mathrm{mM}$ was then added to oxidise TPL-H to TPL and the EPR signal was measured again.

\section{Results}

Figure 1 displays the results obtained with the control group $(n=21$, treated with saline alone) and three experimental groups $(n=11$, treated with TPL; $n=12$, treated with TPL-H; and $n=$ 6 , treated with TPL at the end of ischaemia), each undergoing an identical protocol of ischaemia and reperfusion. The mean (SE) PAH plasma to lumen clearance over eight periods under evaluation (one pre-ischaemia, two ischaemic periods, and five consecutive five minute periods of reperfusion) are depicted in fig 1 .

The intestinal permeability of untreated rats (fig 1, open circles) before and during the ischaemic stage was normally low and did not differ from that of nitroxide treated rats. The intestinal injury, as reflected by mucosal permeability, appeared only in the reperfusion period. The rate of $\mathrm{PAH}$ plasma to lumen clearance then increased significantly compared with its value during the pre-ischaemia and ischaemia periods. The increase in rate was rapid, achieving a five- to sixfold higher level within several minutes. The clearance rate in all experimental groups showed a similar pattern over the post-ischaemic re-flow periods. The increase in PAH plasma to lumen clearance during the reperfusion period was significantly attenuated in rats pretreated with either TPL or TPL-H in comparison to the control group $(p<0.05)$. As seen in fig 1 , the protective effect of TPL (fig 1, squares) was greater than that observed when the rats were pretreated with TPL-H (fig 1, triangles). Pretreatment with TPL significantly decreased the rate of PAH clearance also when the drug was given immediately before reperfusion (fig 1 , closed circles).

TPL/TPL-H EXCHANGE

The fate of nitroxides in isolated cells, organs, and whole animals is important for those who seek to employ nitroxides as contrast agents in MRI or use them as biophysical markers. It was therefore extensively investigated by Swartz et $a l$ as well as by other groups. ${ }^{32} 33$ Their results demonstrated conclusively that the decay of the EPR signal was attributable almost exclusively to a reduction to the respective hydroxylamine, and that the signal could be restored using a 1-electron oxidant such as ferricyanide. The paramagnetic nature of TPL allows convenient monitoring of its distribution and persistence in the rat. During ischaemia and reperfusion stages, samples of blood and perfusate were taken from rats treated with TPL and the level of the residual nitroxide was determined using EPR spectrometry as detailed in the Methods section. $\mathrm{K}_{3} \mathrm{Fe}(\mathrm{CN})_{6} 1 \mathrm{mM}$ was then added to oxidise TPL-H and the intensity of the EPR signal of TPL was measured again. As seen in fig $2 \mathrm{~A}$, more than $50 \%$ of the nitroxide in the blood was reduced to hydroxylamine. In the perfusate the hydroxylamine consisted of more than $90 \%$. Similar distributions were observed for rats treated with TPL-H rather than with TPL (fig 2B).

\section{Discussion}

Intestinal ischaemia is a clinical phenomenon affecting humans of all ages, especially the old 
and the very young. ${ }^{3}$ The clinical situations in which intestinal ischaemia is involved are numerous and include acute mesenteric ischaemia, intestinal obstruction, incarcerated hernia, mid-gut volvulus and necrotising enterocolitis. ${ }^{2122}$ Intestinal ischaemia not only affects the bowel but has severe remote effects on other organs such as the lungs, ${ }^{23}$ heart, ${ }^{24}$ and liver. ${ }^{25}$ There is a large body of evidence showing that one of the earliest changes in intestinal mucosa in response to $I / R$ injury is increased mucosal permeability ${ }^{6}$ and that this strongly correlates to histological assessment of mucosal injury. ${ }^{26}$ The increased mucosal permeability can be quantitated to serve as an accurate measure for assessing different treatment modalities aimed at the reduction of $I / R$ injury. A commonly used method for quantitating mucosal permeability is by measuring the rate of mucosal to lumen clearance. ${ }^{11}{ }^{27}$ This indicator of mucosal permeability provides a quantitative, objective, reproducible, and convenient measurement of mucosal integrity. Clearance measurements can also be performed continuously throughout the experiment, thus providing a moment-to-moment monitoring of mucosal permeability.

\section{THE THERAPEUTIC WINDOW}

Nitroxides are known to protect only when present during the injurious processes but no evidence was found indicating any repair activity. The partition of nitroxides inside the cells is practically instantaneous and fig 1 shows indeed that TPL also diminished the $\mathrm{I} / \mathrm{R}$ injury when given immediatley before reperfusion. This indicates that nitroxides predominantly affect the reperfusion component of the damage. It also further substantiates the conclusion that the injurious process starts with reperfusion. While the TPL/TPL-H couple does not lose its efficacy when given before the ischaemic stage (fig 1), a pretreatment stage is not essential for the antioxidative activity of nitroxide which demonstrates the practical aspect of this potential drug.

THE ROLE OF NITROXIDE

The antioxidative activity of nitroxide has been previously demonstrated against various insults in diverse experimental model systems. The present results demonstrate, for the first time, the potential use of nitroxides against I/R injury. The protective effect of TPL/TPL-H against $\mathrm{I} / \mathrm{R}$ injury was evident by the decrease in the intestinal permeability. Since TPL reacts predominantly with paramagnetic species, its effect further substantiates the involvement of ROS in mediating the I/R intestinal injury. Nitroxides can operate through several mechanisms including catalytic dismutation of $\mathrm{O}_{2}^{-28}{ }^{29}$ :

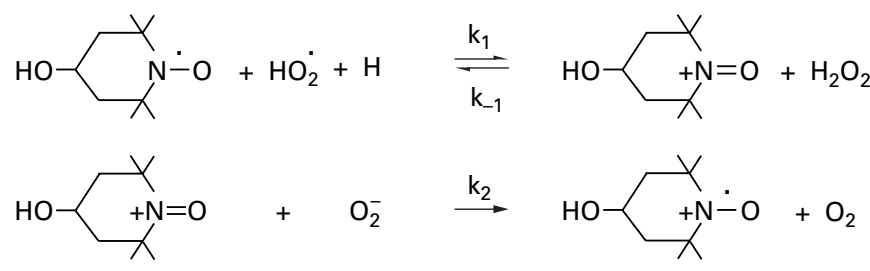

Because nitroxides effectively remove both extracellular and intracellular superoxide, they are anticipated also to affect the concentration of NO. Since superoxide radicals readily react with NO to form peroxynitrite, the catalytic removal of superoxide indirectly elevates the steady state. It was also previously suggested that nitroxides pre-empt the Fenton reaction by oxidising redox active transition metals associated with free radical induced damage. ${ }^{30}$ More recently, nitroxides were shown to catalytically facilitate catalase-like activity of heme proteins and to detoxify their ferryl species. ${ }^{31}$ However, while the present results establish the protective activity of nitroxides against I/R injury, they do not allow a clear distinction among the various potential mechanisms underlying the protection.

Inside the body both the nitroxide and the hydroxylamine coexist. As seen in fig 2, the distribution between TPL and TPL-H was independent of whether TPL or TPL-H was administered to the rats. The metabolism of nitroxides in biological systems has previously been studied extensively. ${ }^{32}$ Nitroxides in tissues are primarily, though not exclusively, ${ }^{33}$ reduced through enzyme associated mechanisms. This bioreduction, which predominantly occurs intracellularly in the mitochondria yielding almost exclusively the respective hydroxylamines, is rapid. ${ }^{32}$ The oxidation of hydroxylamine to nitroxide can also occur at high rates, ${ }^{345}$ and with some nitroxides can be comparable to the rate of nitroxide reduction. This can explain the rapid formation of nitroxide upon administration of the hydroxylamine. Non-enzymatic processess also contribute to TPL-H oxidation and recently it was suggested that the hydroxylamine and oxo-ammonium cation might comproportionate, yielding two nitroxide molecules. ${ }^{36}$

Since both TPL and its reduced form TPL-H might confer protection, ${ }^{37}$ the rapid metabolic exchange limits the distinction between the protective activities of the two forms. Nevertheless, treating the rats with TPL had a greater effect than treating them with TPL-H. Since the nitroxide, unlike its reduced form, can oxidise reduced metals, the results suggest that this mode of action primarily contributes towards the protective effect. However, this observation does not exclude the possibility that the hydroxylamine per se might possess protective activity. Moreover, through the continuous exchange between the two forms they can act as self-replenishing antioxidants that thus possess catalytic protective activity. This feature indicates the potential of this unique class of antioxidants against oxidative stress in general, and I/R injury in particular.

The authors thank Mrs Carol Levi for her skillful technical assistance. This research was partly supported (AS) by a grant from the Israeli Ministry of Health.

1 Scholz FJ. Ischemic bowel disease. Radiol Clin North Am 1993;31:1197-218.

2 Jrvinen O, Laurikka J, Salenius JP, et al. Acute intestinal ischaemia. A review of 214 cases. Ann Chir Gynaecol 1994; ischaemia.

3 Haglund U. Gut ischaemia. Gut 1994;35:573-6. 
4 Cunningham CG, Reilly LM, Stoney R. Chronic visceral ischemia. Surg Clin North Am 1992;72:231-44.

5 Amoury RA. Necrotizing enterocolitis: a continuing problem in the neonate. World F Surg 1993;17:363-73.

6 Zimmerman BJ, Granger DN. Reperfusion injury. Surg Clin North Am 1992;72:65-83.

7 Mitsudo S, Brandt LJ. Pathology of intestinal ischemia. Surg Clin North Am 1992;72:43-63.

8 Grisham MB, Benoit JN, Granger DN. Assessment of leukocyte involvement during ischemia and reperfusion of intestine. Methods Enzymol 1990;186:729-42.

9 Crissinger KD, Kvietys PR, Granger DN. Pathophysiology of gastrointestinal mucosal permeability. F Intern Med Suppl 1990;732:145-54

10 Cassutto $\mathrm{BH}$, Misra HP, Pfeiffer CJ. Intestinal postischemic reperfusion injury: studies with neonatal necrotizing enterocolitis. Acta Physiol Hung 1989;73:363-9.

11 Langer JC, Sohal SS, Riddell RH. Mucosal permeability to 51Cr EDTA following subclinical intestinal ischemia$51 \mathrm{Cr}$ EDTA following subclinical intestinal ischemia-
reperfusion injury in the weanling rat. $\mathcal{F}$ Pediatr Surg 1993; 28:601-5.

12 Langer JC, Sohal SS, Blennerhassett P. Mucosal permeability after subclinical intestinal ischemia-reperfusion injury: an exploration of possible mechanisms. I Pediatr Surg 1995;30:568-72.

13 Bhatia AM, Feddersen RM, Musemeche CA. The role of luminal nutrients in intestinal injury from mesenteric reperfusion and platelet-activating factor in the developing rat. F Surg Res 1996;63:152-6.

14 Pitt RM, McKelvey TG, Saenger JS, et al. A tungstensupplemented diet delivered by transplacental and breastfeeding routes lowers intestinal xanthine oxidase activity and affords cytoprotection in ischemia-reperfusion injury to the small intestine. F Pediatr Surg 1991;26:930-5.

15 Krasna IH, Lee RT. Allopurinol protects the bowel from necrosis caused by indomethacin and temporary intestinal ischemia in mice. F Pediatr Surg 1993;28:1175-7.

16 Rachmilewitz D, Karmeli F, Okon E, et al. A novel antiulcerogenic stable radical prevents gastric mucosal antiulcerogenic stable radical prevent

17 Karmeli F, Eliakim R, Okon E, et al. A stable nitroxide radical effectively decreases mucosal damage in experimental colitis. Gut 1995;37:386-93.

18 Beit-Yannai E, Zhang R, Trembovler V, et al. Cerebroprotective effect of stable nitroxide radicals in closed head injury in the rat. Brain Res 1996;717:22-8.

19 Bitterman N, Samuni A. Nitroxide stable radicals protect aganst hyperoxic-induced seizures in rats. Undersea Hyperb Med 1995;22(suppl):47-8. 20 Brun C. A rapid method for the determination of Clin Med 1951;37:955-8.
21 Caplan MS, MacKendrick W. Necrotizing enterocolitis: a review of pathogenetic mechanisms and implications for review of pathogenetic mechanisms and i
prevention. Pediatr Pathol 1993;13:357-69.

22 Jaile JC, Levin T, Wung JT, et al. Necrotizing enterocolitis in infancy. Surg Clin North Am 1992;72:261-76.

23 Carden DL, Young JA, Granger DN. Pulmonary microvascular injury after intestinal ischemia-reperfusion: role of P-selectin. F Appl Physiol 1993;75:2529-34.

24 Horton JW, White DJ. Free radical scavengers prevent intesinal ischemia-reperfusion-mediated cardiac dysfunction. $f$ Surg Res 1993;55:282-9.

25 Poggetti RS, Moore EE, Moore FA, et al. Gut ischemia/ reperfusion-induced liver dysfunction occurs despite susreperfusion-induced liver dysfunction occurs despite sus-
tained oxygen consumption. F Surg Res 1992;52:436-42.

26 Parks DA, Bulkley GB, Granger DN, et al. Ischemic injury in the cat small intestine: role of superoxide radicals. Gastroenterology 1982;82:9-15.

27 Sibbons P, Spitz L, van Velzen D. Increased mucosal permeability after intestinal ischemia-reperfusion injury is mediated by local tissue factors. F Pediatr Surg 1992;27:329-31.

28 Krishna MC, Grahame DA, Samuni A, et al. Oxoammonium cation intermediate in the nitroxide-catalyzed dismutation of superoxide. Proc Natl Acad Sci USA 1992;89. $5537-41$.

29 Krishna MC, Russo A, Mitchell JB, et al. Do nitroxide antioxidants act as scavengers of $\mathrm{O} 2-$ or as SOD mimics? $\mathrm{F}$ Biol Chem 1996;271:26026-31.

30 Mitchell JB, Samuni A, Krishna MC, et al. Biologically active metal-independent superoxide dismutase mimics. Biochemistry 1990;29:2802-7.

31 Krishna MC, Samuni A, Taira J, et al. Stimulation by nitroxides of catalase-like activity of hemeproteins. Kinetics and mechanism. F Biol Chem 1996;271:26018-25.

32 Swartz HM. Principles of the metabolism of nitroxides and their implications for spin trapping. Free Radic Res Commun 1990;9:399-405.

33 Belkin S, Mehlhorn RJ, Hideg K, et al. Reduction and destruction rates of nitroxide spin probes. Arch Biochem Biophys 1987;256:232-43.

34 Chen K, Swartz HM. Oxidation of hydroxylamines to nitroxide spin labels in living cells. Biochim Biophys Acta 1988:970:270-7.

35 Chen K, Glockner JF, Morse P, et al. Effects of oxygen on the metabolism of nitroxide spin labels in cells. Biochemistry 1989;28:2496-501.

36 Zhang R, Pinson A, Samuni A. Both hydroxylamine and nitroxide protect cardiomyocyte from oxidative stress. Free Radic Biol Med (in press)

37 Nilsson UA, Olsson LI, Carlin G, et al. Inhibition of lipid peroxidation by spin labels. Relationships between structure and function. F Biol Chem 1989;264:11131-5. 